\title{
Chapter 19 \\ Context Matters: GIS-Based Spatial Analysis of the Nasca Geoglyphs of Palpa
}

\author{
Karsten Lambers and Martin Sauerbier
}

\begin{abstract}
In this chapter we report on the GIS-based analysis of the Nasca geoglyphs of Palpa, Peru, undertaken in the course of the Nasca-Palpa Archaeological Project. We focus here on the analysis of spatial relationships between the geoglyphs and the surrounding landscape in terms of visibility and orientation. Our motivation for this contextual analysis was to gain a better understanding of the function and meaning of the geoglyphs by virtually assuming the viewpoints of the people who conceived, built, and used the geoglyphs between approximately $400 \mathrm{BC}$ and $800 \mathrm{AD}$. In this sense our study of geoglyph visibility and orientation is a contribution to current attempts to incorporate cultural variables into the quantitative environment of GIS, thereby rendering GIS a more useful instrument for archaeological research. This approach required the development of new GIS tools tailored to the specific needs of archaeological analysis. The results of our study indicate that the geoglyphs can be understood as stages for public rites performed by social groups, whereas the incorporation of the surrounding landscape through visual links was apparently not a major concern.
\end{abstract}

\subsection{GIS Applications in Archaeology: Chances and Limitations}

Geographical information systems (GIS) have become a widespread tool in archaeological research due to their manifold capabilities in terms of data capture, management, analysis, and visualisation (Wheatley and Gillings 2002; Conolly and Lake 2006). Because of their origin in cartography and geography, GIS are especially well suited for archaeological investigations at a regional scale, be they more traditional studies of settlement patterns or recent approaches to landscape archaeology. However, as GIS were not originally

K. Lambers $(\square)$

University of Konstanz, Zukunftskolleg, Department of Computer Science,

P.O. Box 697, 78457 Konstanz, Germany

e-mail: karsten.lambers@uni-konstanz.de 
conceived for archaeological applications, their usability in archaeology is affected by certain conceptual limitations. The most important constraint results from the fact that GIS are designed to handle measurable data that can easily be quantified and expressed by either of the two standard data formats of GIS, vector and raster data, whereas other kinds of information are difficult to process and analyse. Due to this requirement, data describing the current environment that can be obtained through a variety of methods and sensors are the most readily available information to be used for GIS-based analyses. This includes, for example, topographical data (digital elevation models), environmental data (hydrological, geological, botanical data), cadastral and administrative data (borders, real estate ownership), and economic data (land and resource use).

Archaeological information, on the other hand, often differs considerably from the kinds of data mentioned above. It is impossible to record archaeological data with the degree of reliability, completeness, and accuracy of common environmental or cadastral datasets. For example, archaeological sites and features tend to be wholly or partially destroyed, considerably altered, or buried, such that a complete inventory of the material remains of a given society under study is impossible to achieve. Archaeological data furthermore describe material remains of past societies that in most cases experienced a quite different environment from the one modelled by modern geodata, inasmuch as climatic conditions, land use, population density, and other parameters have often changed considerably since the time period under study. This leads to a general problem of archaeological GIS applications, namely that datasets that are ultimately incompatible in terms of their quality and time reference are commonly related to each other and analysed in conjunction. A careful, casespecific consideration of which aspects of available environmental and archaeological data actually represent the situation at the time under study is required to mitigate this problem.

A related major problem of using GIS in archaeological research concerns the existence of many parameters operational in the cultural development of a society over time that are not easy to capture and translate into GIS-compatible data. Such parameters refer to qualitative information that is difficult to measure, quantify, or georeference. This includes the significance or value of areas and places - for example, areas of high or low prestige, sacred places, or places of remembrance - as well as the often unmarked boundaries of political, ethnic, religious, or linguistic spheres. Although spatially reflected, parameters such as these are often ambiguous, ephemeral, or contested.

They are therefore difficult to model in the abstract, quantitative, Cartesian framework provided by GIS. Rather, they correspond to cultural, social, and qualitative concepts of space that today are often subsumed under the term 'landscape' (for a comprehensive discussion see Anschuetz et al. (2001) and Gramsch (2003); cp. Palang and Fry (2003)). In this conceptual framework, the environment provides the spatial framework for many different landscapes that depend on subjective, individual, situational, and a variety of other perspectives 
that may be difficult to model in GIS. This has led to serious concerns about the usefulness of GIS in archaeological research: 'Given [the] apparent incompatibility between recent theoretical perspectives on landscape and space, and the nature of GIS, is the latter really a suitable environment for the interpretative analysis of archaeological data?' (Witcher 1999:15).

As a contribution to this debate, we focus here on the analysis of the visibility and orientation of archaeological features, in this case the Nasca geoglyphs of Palpa described in the following section. Over the past decade, visibility and observation have been extensively studied in archaeological GIS applications by using viewshed analysis for elevation-dependent visibility studies and visualisation and virtual reality for reconstructing possible observations (see discussion in Wheatley and Gillings (2000) and Lake and Woodman (2003)). In visibility analysis, variables of the physical environment such as terrain elevation and Euclidean distance serve as spatial proxies to study cognitive and other phenomena that are not directly measurable, such as perception (Whitley 2004). Such studies start from a middle ground between 'the applied scientism of processual archaeology and the attempted humanism of post-processual approaches' in landscape archaeology (Lock and Harris 2006:43). Addressing the concerns raised above, visibility analysis is thus a pragmatic attempt to indirectly approach qualitative cultural phenomena within the quantitative and Cartesian framework of GIS (cp. Verhagen et al. 2007). Specifically, it allows us to reconstruct how archaeological features may have been perceived by people moving through the study area, and how they might have been spatially related to cultural and natural components of their surrounding environment. In the case of the Nasca geoglyphs of Palpa, this evidence is hoped to provide clues on the function and meaning of the geoglyphs.

\subsection{Studying the Visibility and Orientation of the Nasca Geoglyphs in Palpa}

The object of our GIS-based spatial analysis was the geoglyphs of Palpa, in the northern part of the Río Grande basin, where a long-term multidisciplinary research project to investigate the cultural and environmental history of the Nasca region has been conducted since 1997 (Reindel and Wagner this volume). The pampas of Nasca farther to the south between Río Ingenio and Río Nasca, where the best-known concentration of geoglyphs is located, have recently been included in our study area, and the topography of this area has been recorded with advanced methods of digital photogrammetry (Sauerbier this volume). However, although intended for the future, a GIS-based archaeological study of the geoglyphs on the pampas of Nasca has not yet been undertaken and is thus not covered here.

Built approximately between $400 \mathrm{BC}$ and $800 \mathrm{AD}$, the geoglyphs cover the stony desert surface of the hills and pampas between the fertile river oases of the 
Río Grande basin at the foot of the Andes (Kern and Reiche 1974; Aveni 1990; 2000a,b). Although biomorphic figures are today the best-known geoglyphs, they are by far outnumbered by geometric shapes such as lines, trapezoids, and spirals, many of which overlap, converge, or are grouped into huge complexes that often emerged over several centuries (Fig. 19.1). The origin of the geoglyph phenomenon can be traced back to the petroglyph tradition of the Paracas culture of the Early Horizon ( 800 to $200 \mathrm{BC}$ ), however, the vast majority of geoglyphs that are visible today were made during the Nasca culture of the Early Intermediate period (200 BC-650 AD; Lambers 2006b).

Through the geoglyphs, the ancient inhabitants of the Río Grande basin marked and transformed the desert landscape in a unique and spectacular way that can still be appreciated today. Whatever their specific meaning, the geoglyphs clearly integrated the vast and uninhabitable desert plains into the cultural domain of the valley-based society (Silverman 1990: 451). In spite of the wide variety of available literature on the origin and function of the geoglyphs, archaeological investigations have been surprisingly sparse (see review in Aveni 1990). The principal objective of our investigation of the Palpa geoglyphs was therefore to learn more about the geoglyphs through a thorough field study of the geoglyphs and their associated features and artefacts (Reindel et al. 2003). To this end, we started with a comprehensive digital mapping of the geoglyphs (Sauerbier and Lambers 2003, 2004) that

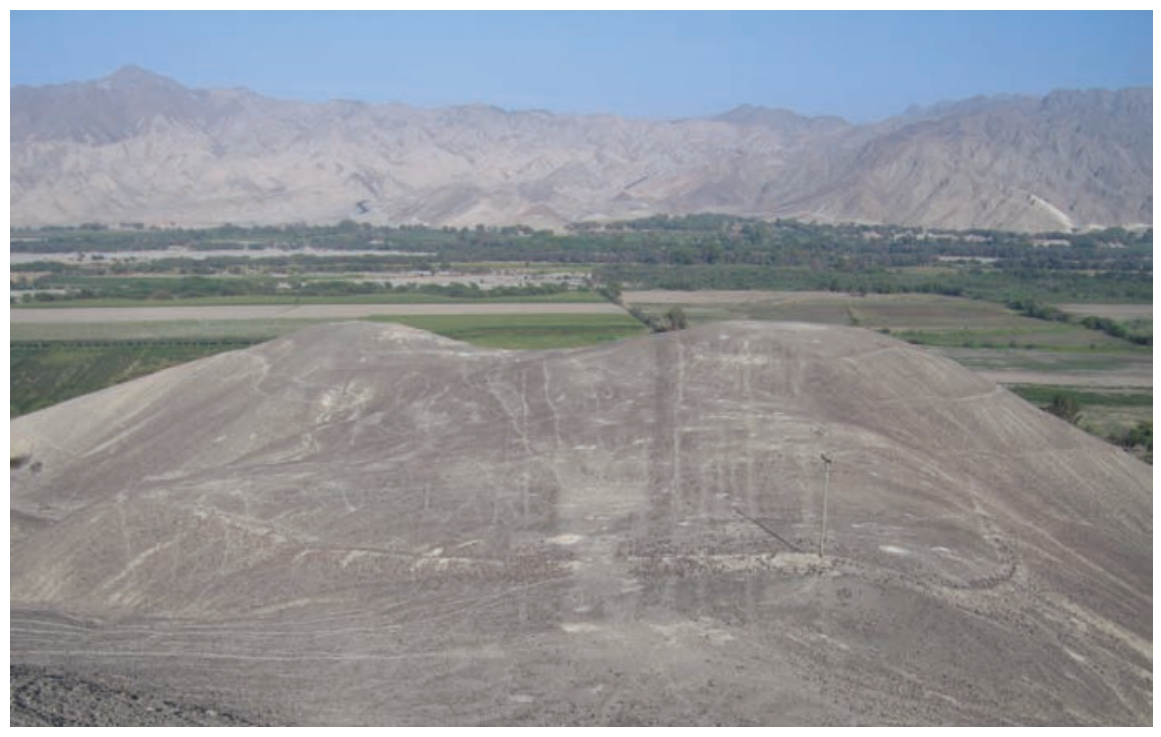

Fig. 19.1 A trapezoid and several linear geoglyphs on a spur overlooking the Río Grande valley westwards (site PV66-122). A range of hills is visible in the background. (Photo: K. Lambers) 
enabled the first GIS-based spatial analysis of the geoglyphs in their natural and cultural context.

Archaeological fieldwork on geoglyph sites in Palpa revealed ample traces of ancient activity on and around geoglyphs such as placement of ceramic vessels and other offerings along geoglyph borders, frequent walking over linear geoglyphs, and a variety of acts associated with stone platforms and wooden posts erected on trapezoidal geoglyphs (Lambers 2006b; Reindel et al. 2006). The background of these activities can to a certain degree be inferred from the archaeological evidence. Although offerings such as Spondylus shells, crawfish, and field crops strongly hint at a cult revolving around concepts of water, irrigation, and fertility, rites performed on geoglyph sites had at the same time probably an important social dimension concerning the groups involved, as discussed below.

Furthermore, evidence from unfinished geoglyphs indicates that the construction and remodelling of geoglyphs and geoglyph sites was a continuous process that was arguably never regarded as finished. This process apparently involved large parts of the ancient population and had a meaning in its own right, being likely as important as the actual use of the geoglyphs for other forms of group activity, with which it was closely interwoven. Thus, whatever their intended meaning, the archaeological evidence clearly indicates that geoglyphs served as locations for continuous, structured, and varied group activity.

This archaeological finding is in stark contrast to today's perception of the geoglyphs. Modern visitors to the Nasca region tend to get the impression that geoglyphs are only visible from the air. This false notion perpetuated through popular media is fostered by modern patterns of movement through the region that are very distinct from ancient ones. Today, visitors to Nasca spend their time mainly in one of the valleys without ever entering the pampas. To see the geoglyphs, they board small airplanes that offer spectacular views of the pampas from above. Due to this remote perspective, geoglyphs are today often seen and interpreted as images or pictures largely devoid of any context. Labellings of well-known biomorphic geoglyphs such as Monkey, Spider, or Lizard are hardly disputable due to their distinct shape, however, popular names of geometric geoglyphs such as Yarn and Needle, Sundial, or Paddle Wheel (Kern and Reiche 1974; Aveni 2000a) show that these geoglyphs are often understood as images of real-life objects as well. This modern perspective was not shared by the people who conceived, built, and used the geoglyphs.

A ground-based perspective is therefore a better approach to learn more about the geoglyphs. A close-up view may reveal the size, composition, and construction details of a geoglyph, showing, for example, that many geoglyphs are less straight or clear-cut than they seem from above, and often incorporate elements dating from different time periods. A ground-based perspective also reveals how geoglyphs appear in their topographic setting. This is important, as the landscape in which the geoglyphs are situated may have been incorporated into geoglyph complexes by means of visual links or other kinds of spatial relationships. An investigation of such a possible spatial order may thus 
reveal, in addition to findings from archaeological fieldwork, important clues to understanding the function and meaning of the geoglyphs.

GIS provide tools to simulate such perspectives in a computer environment. For our investigation of the visibility and orientation of the Nasca geoglyphs of Palpa we took into account the issues concerning GIS applications in archaeology mentioned in the previous section. We regard visibility (encompassing vision and observation) as a constitutive yet insufficient element of perception (cp. Witcher 1999:14 and Wheatley and Gillings 2000: 3f), thus allowing us to approach perspectives of the people who conceived, built, and used the geoglyphs in order to learn more about their function and meaning. We developed new tools for the calculation of geoglyph orientation, and adapted and enhanced proven methods such as cumulative viewshed calculation (Wheatley 1995; Lake et al. 1998) for visibility analysis. We are aware that advanced methods and concepts such as Higuchi viewsheds (Wheatley and Gillings 2000) and visual affordance and prominence (Llobera 1996, 2001, 2003) might be fruitful approaches to further explore the geoglyphs in the future.

For our investigations in the Palpa region we relied heavily on the results of recent investigations into the geomorphology and paleoclimate of the study area (Eitel and Mächtle this volume) in order to determine which elements of the present-day landscape reflected in the geodata available for our study were representative of the time when the geoglyphs were built and used. Considering the setting of the geoglyphs on desert plains surrounded by river valleys, a coastal cordillera to the west, and the Andes to the east, an investigation of two different perspectives seemed worthwhile: the view of the geoglyphs, and the reverse view from the geoglyphs on the surrounding landscape. This bidirectional visibility study was aimed to detect possible recurrent patterns of spatial relationships between archaeological features and the surrounding landscape.

\subsubsection{The View from Outside: Visibility of the Geoglyphs}

In Late Paracas and Nasca times, the desert between the fertile river valleys was well integrated into the daily life of the ancient society. People left their settlements in the valleys on a regular basis, ascending the sandy slopes that led up to the flat plains, crossing plateaus and dry valleys in order to spend time at geoglyph sites for all kinds of group activity (Aveni 1990; Reinhard 1996; Lambers 2006b). People thus frequently assumed viewpoints that today are largely abandoned. From many of these vantage points, the geoglyphs, the groups of people gathering upon them, and the rites performed on them, were an important visual element of the landscape. In this sense, it seems possible that this activity taking place on geoglyph sites was meant to be seen by others. Although the sociopolitical organisation of Nasca society is still a matter of debate, it may have been organised in large clanlike groups (Lambers 2006b: 119ff). These groups may have been associated with major geoglyph complexes, 
which they developed over time and used for group rituals. In this hypothetical scenario, rites performed on geoglyph sites may have served to raise awareness of group identitiy among group members as well as observers. This would have required good visibility of geoglyph sites.

In order to test the hypothesis that geoglyphs were deliberately placed in locations that afforded good visibility, we decided to compare the visibility of points on geoglyph sites to the visibility of points randomly distributed over the terrain. If the visibility of the geoglyph points were significantly better, this would support our hypothesis. While the details of this study have been presented elsewhere (Lambers and Sauerbier 2006, 2008), in this section we focus on the general outline of our investigation and summarise its main results.

The situation in Palpa provides quite favourable conditions for GIS-based visibility studies, as several factors often cited as questioning the results of such simulations are of limited relevance here. Most importantly, geomorphological change that occurred since Nasca times has been minimal, at least concerning the desert portion of the landscape, as indicated by recent geoarchaeological research (Eitel et al. 2005; Mächtle et al. 2006; Mächtle 2007; Eitel and Mächtle this volume). This means that digital terrain models (DTMs) modelling the current state of the topography can be regarded as largely representative of the conditions in Nasca times at least with respect to the pampas and surrounding mountains. Furthermore, vegetation cover (Llobera 2007) does not pose a problem in a desert environment. Vegetation cover is, and used to be, present in varying density on the flood plain, however, it may only marginally, if at all, have obstructed intervisibility between the pampas and the surrounding mountains, as both are situated on higher elevations.

The question of discernability of cultural features over large distances (Ogburn 2006) is more difficult to answer. As shallow surface markings, geoglyphs may not have been visible beyond a certain distance, which is confirmed by our experience from eight months of fieldwork on geoglyph sites around Palpa, even though geoglyph outlines and their colour contrast to the surrounding desert surface must have been much clearer in Nasca times. However, considering the ample evidence of group activity on geoglyph sites, it is highly likely that it was not the geoglyphs themselves, but rather people interacting upon and moving over them that were at the focus of attention in ancient times. We know from our own experience that single persons in the desert are distinguishable as moving dark spots over a distance of several kilometres, even across the wide flood plain of the Palpa and Viscas rivers. Groups of people must have been visible even more clearly. Therefore, discernability limitations over large distances are again not a major problem affecting the study of geoglyph visibility.

For our investigation we had at our disposal a DTM of the study area generated from aerial and satellite images with $30 \mathrm{~m}$ resolution and an accuracy of $18.7 \mathrm{~m}$ with respect to a manually measured DTM derived purely from aerial images (Sauerbier and Lambers 2003; Sauerbier et al. 2006). The DTM was large enough to avoid edge effects when calculating visibility. We defined every centre point of a DTM cell intersected by a geoglyph as a geoglyph point, 
resulting in 2067 geoglyph points. We then determined visibility values for each of these points by calculating lines of sight (LoS) between each DTM cell and each geoglyph point, taking into account observer's height, earth curvature, and refraction. The resulting values indicated for each geoglyph point from how many DTM cells it was visible.

In order to assess these values, we calculated a second sample of 2067 points in Matlab that were randomly distributed over our study area. For these random points we calculated visibility values by the same method as for the geoglyph points. By doing so, we obtained a reference dataset to which we compared the visibility values of the geoglyph points. Applying the two-sample KolmogorovSmirnov goodness-of-fit test, we were able to determine that the visibility of the geoglyph points differed significantly from the visibility of the random points (Fig. 19.2). The corresponding chart in our detailed report on this study (Lambers and Sauerbier 2008, Fig. 8) shows miscalculated values, even though the values given in the text are correct. We thank Jens Andresen and Irmela Herzog for kindly pointing out this mistake to us, and correct the chart here (Fig. 19.2).

A direct comparison of the visibility values showed that a majority of geoglyph points reached much higher values than the random points (Lambers and Sauerbier 2008; Fig. 7). At the same time, our study showed that the variable visibility was largely independent of other spatial variables, such as slope degree and elevation. This allowed us to conclude that visibility had had an effect on the choice of place for geoglyphs in that locations that were well visible from the surrounding landscape were preferred. This result clearly supports our hypthesis that rites performed by social groups on geoglyphs were meant to be seen, thus allowing glimpses into the social dynamics of ancient Nasca society.

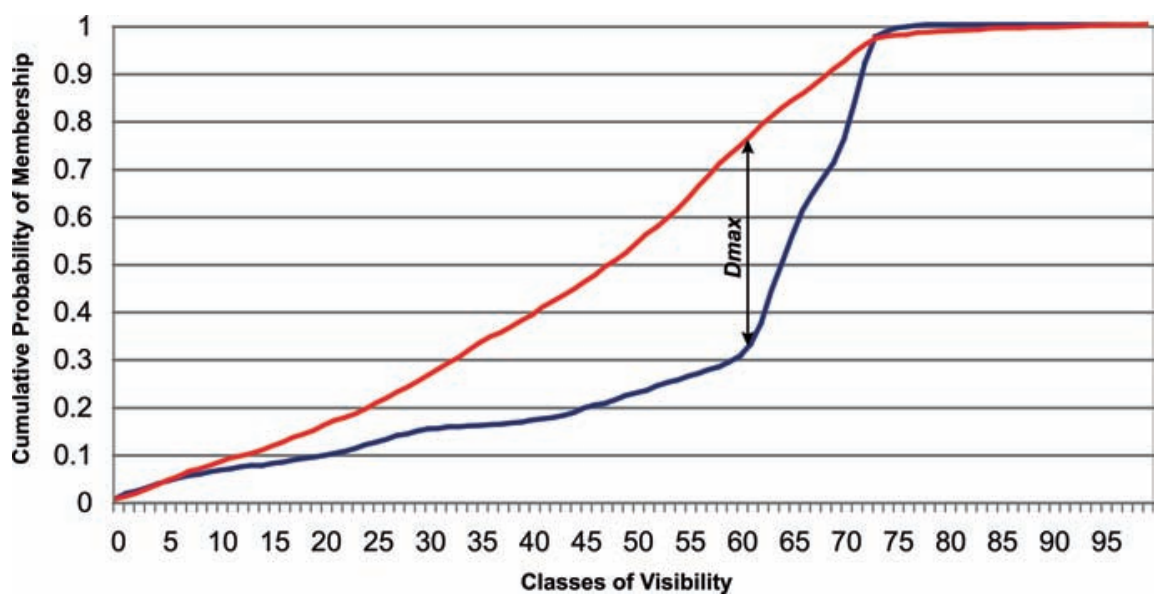

Fig. 19.2 Cumulative probability of membership in visibility classes of points on geoglyphs (blue line) versus random points (red line). Dmax indicates the maximum difference between both curves. (For further detail see Lambers and Sauerbier 2008) 


\subsubsection{The View from Within: Orientation of the Geoglyphs}

From our study of perspectives of the geoglyphs we turned to a study of perspectives from the geoglyphs, the results of which are presented in detail in this section. While apparently meant to be observed by others, rites performed on geoglyph sites at the same time afforded the participants manifold views of the surrounding landscape. As geoglyphs visually marked and structured the desert surface, and often assumed geometric shapes with predominant straight elements such as borders or axes of trapezoids, straight sections of lines, and so on, these straight elements may have served as visual pointers to certain natural or cultural features of the surrounding landscape.

Geoglyph orientation has up to now usually been discussed in terms of astronomical alignments of geoglyphs with the position of celestial bodies (Hawkins 1974; Aveni 1990; Reiche 1993; Teichert 2007). As the astronomical hypothesis had been extensively tested and failed to provide a plausible explanation for the majority of known geoglyphs, we decided to focus our investigation of geoglyph orientation in Palpa on other possible targets closer by. According to well-documented Andean traditions, mountains may have been regarded in the Nasca region as seats of deities and sources of water (Rostworowski 1993; Reinhard 1996). Because many offerings placed along geoglyphs and on stone platforms indicate a concern for water, a link between geoglyphs and mountain veneration seemed possible. Visually dominant straight sections of certain geoglyphs may thus have directed the views of people gathering upon them to certain mountains. Another observation hints in this direction as well. The well-documented line centres on the pampas, from which straight lines radiate out or in which they converge, respectively, tend to be located on elevated terrain such as low hills or rock outcrops (Aveni 1990; Reiche 1993). This supports the idea of linear geoglyphs pointing towards terrain peaks. That way, lines would visually incorporate elements of the surrounding landscape into the rites performed on geoglyph sites.

In order to test this hypothesis, we first needed to determine the orientation axes of the Palpa geoglyphs. These were calculated on the basis of the digital documentation of geoglyph outlines (Sauerbier and Lambers 2004) that had resulted in two sets of $3 \mathrm{D}$ vector data: polylines mapping the preserved borders of geoglyphs usually marked by heaped stones, and polygons covering the most likely original cleared area of a given geoglyph, defined by combining and complementing the polylines. On the basis of these polygons, we first calculated the area and the centre of gravity of each geoglyph based on Gauss' theorem, and then derived the azimuth of the principal axes by means of a principal axis transformation:

$$
A_{\text {Geoglyph }}=\frac{1}{2} \sum_{i=1}^{n}\left(x_{i} y_{i+1}-x_{i+1} y_{i}\right)
$$


where $A_{\text {Geoglyph }}$ is the area of a polygon, $n$ the number of vertices, and $x, y$ the vertex coordinates. With the static moments $S_{x}$ and $S_{y}$ in the $x$ - and $y$-direction:

$$
S_{x}=\frac{1}{6} \sum_{i=1}^{n}\left(x_{i} y_{i+1}-x_{i+1} y_{i}\right)\left(y_{i+1}+y_{i}\right)
$$

and

$$
S_{y}=\frac{1}{6} \sum_{i=1}^{n}\left(x_{i} y_{i+1}-x_{i+1} y_{i}\right)\left(x_{i+1}+x_{i}\right)
$$

we obtained the coordinates of the centre of gravity $X_{C G}$ and $Y_{C G}$ as

$$
X_{C G}=\frac{S_{y}}{A_{\text {Geoglyph }}} \quad \text { and } \quad Y_{C G}=\frac{S_{x}}{A_{\text {Geoglyph }}} .
$$

In a next step, we calculated the moments of inertia $I_{u u}, I_{v v}$, and $I_{u v}$ in the principal axis coordinate system:

$$
I_{\alpha \beta}=\int_{A} \rho(\vec{r})\left(r^{2} \partial_{\alpha \beta}-x_{\alpha} x_{\beta}\right) d A
$$

with

$$
\partial_{\alpha \beta}=\left\{\begin{array}{l}
1: \alpha=\beta \\
0: \text { else }
\end{array}, \quad \alpha=u, v, \quad \beta=u, v \quad \text { and } \quad r_{i}=\sqrt{x_{i}^{2}+y_{i}^{2}} .\right.
$$

We then derived the azimuth of each principal axis with respect to the UTM Zone $18 \mathrm{~S}$ system:

$$
\alpha=\frac{1}{2} \arctan \left(\frac{2 I_{u v}}{I_{v v}-I_{u u}}\right) .
$$

Although the method that we used theoretically allows the axis of orientation to be determined for all kinds and shapes of geoglyphs, we limited our calculation to those geoglyphs that actually feature visually dominant straight sections, such as straight and meandering lines, rectangles, and trapezoids (Fig. 19.3), whereas all biomorphic figures, spirals, and the like were not considered. This way, 421 geoglyphs out of a total of 639 defined geoglyphs were considered, slightly more than in our first attempt (Lambers 2006b:116f, Fig. 45).

Compared to previous attempts to determine geoglyph orientation by field measurements, our approach has the advantage of factoring in all the irregularities featured by many geoglyphs which are reflected in the detailed geoglyph 


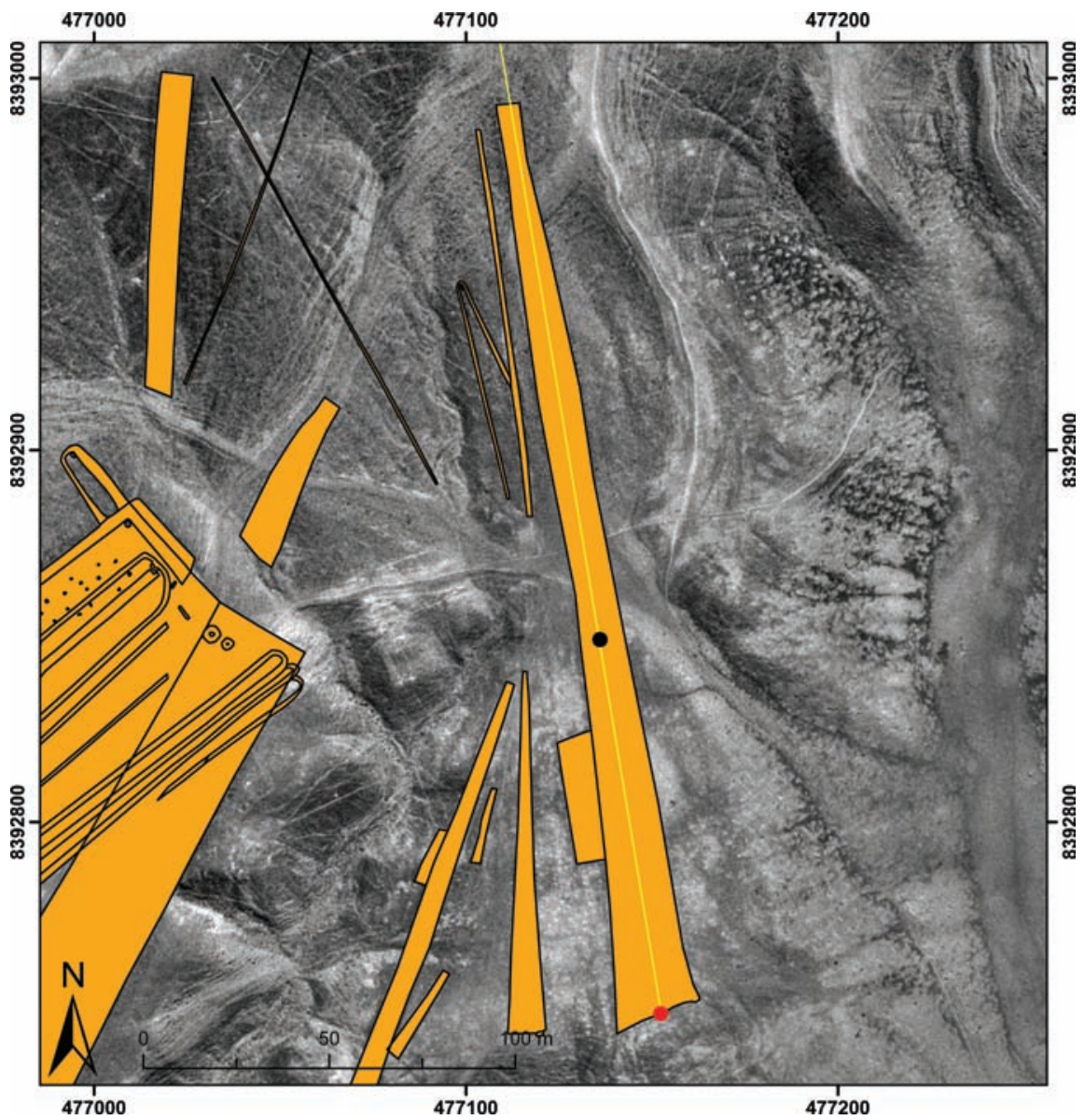

Fig. 19.3 Example of a geoglyph represented by a polygon from which the centre of gravity (black dot) and then the axis of orientation (yellow line) were calculated. The far intersection of the axis with the polygon border as seen from the peak (red dot) serves as observer's point for LoS calculation (cp. Figs. 19.5 and 19.6.) (Coordinates: UTM 18 S, WGS 84)

mapping. Furthermore, for all geoglyphs considered here, the axis of orientation calculated by means of a principal axis transformation runs through the centre of gravity of the geoglyph, as opposed to field measurements that may, for instance, indicate the orientation of one of the two nonparallel borders of a trapezoid instead.

Once the orientations had been established, we needed to test if the corresponding axes intersected mountain peaks, for which we determined local maxima in the terrain elevation of our study area. For this purpose we used the same digital terrain model of the Palpa area described in the previous section. We used an algorithm implemented in the Landserf 2.2 software 
(www.landserf.org) to detect local maxima of terrain elevation in the DTM (Wood 1996: Chap. 5). This algorithm identifies peaks in a raster DTM based on the height differences of each cell with respect to its surrounding neighbour cells. A peak requires a local convexity of the terrain, therefore the necessary and sufficient conditions for a peak can be formulated mathematically by using the first- and second-order derivatives as follows.

$$
\frac{\partial z}{\partial x}=0, \quad \frac{\partial z}{\partial y}=0, \quad \frac{\partial^{2} z}{\partial x^{2}}>0, \quad \text { and } \quad \frac{\partial^{2} z}{\partial y^{2}}>0 .
$$

The size of the window used to search for local maxima can be varied according to the terrain characteristics. In our case, we defined a search window size of $7 \times 7$ cells, although calculations with $5 \times 5$ and $9 \times 9$ cells yielded exactly the same results. A second parameter required for peak detection is the minimum height difference within the search window required to accept a DTM cell as a peak. We decided to set this value to $20 \mathrm{~m}$ in order to detect only significant peaks.

With these parameters, the calculation yielded 116 local maxima for the whole area covered by the DTM (Fig. 19.4). However, 43 out of these were not visible from any of the geoglyphs, as we determined by calculating a multiple viewshed using the geoglyph points described in the previous section as observers' points. Only 73 local maxima were located within the area covered by this viewshed and were thus further considered in our calculation. A review of these 73 points showed that the algorithm had quite reliably identified peaks of mountains, hills, and spurs that we knew from our fieldwork to be visually prominent in our study area.

In the following step we tested which axes of orientation calculated for the geoglyphs intersected these local maxima. In order to account for inaccuracies inherent in the geoglyph mapping as well as in the calculation of both the axes of orientation and the local maxima, a given axis of orientation was regarded as intersecting a local maximum if it passed the actual point within a threshold distance of

$$
\Delta d=\mathrm{d}_{\text {Geoglyph }- \text { Peak }} * \sin (\alpha)+20 \mathrm{~m} \text { with } \alpha=\arcsin \left(0.15 \mathrm{~m} / L_{\text {Geoglyph }}\right),
$$

where $0.15 \mathrm{~m}$ is the accuracy of the photogrammetric measurement of the geoglyph border points, $20 \mathrm{~m}$ the estimated accuracy of the DTM, and $L_{\text {Geoglyph }}$ the length of the orientation axis segment intersecting the geoglyph. The threshold distance $\Delta d$ therefore varies for each possible geoglyph/peak combination, factoring in the accuracy of both the geoglyph mapping and the underlying DTM.

This calculation resulted in 28 orientation axes intersecting a local maximum (Fig. 19.5). Although 50 local maxima were not intersected at all, 19 were intersected by one axis of orientation each, 3 local maxima by two axes each, and one peak even by three axes. 


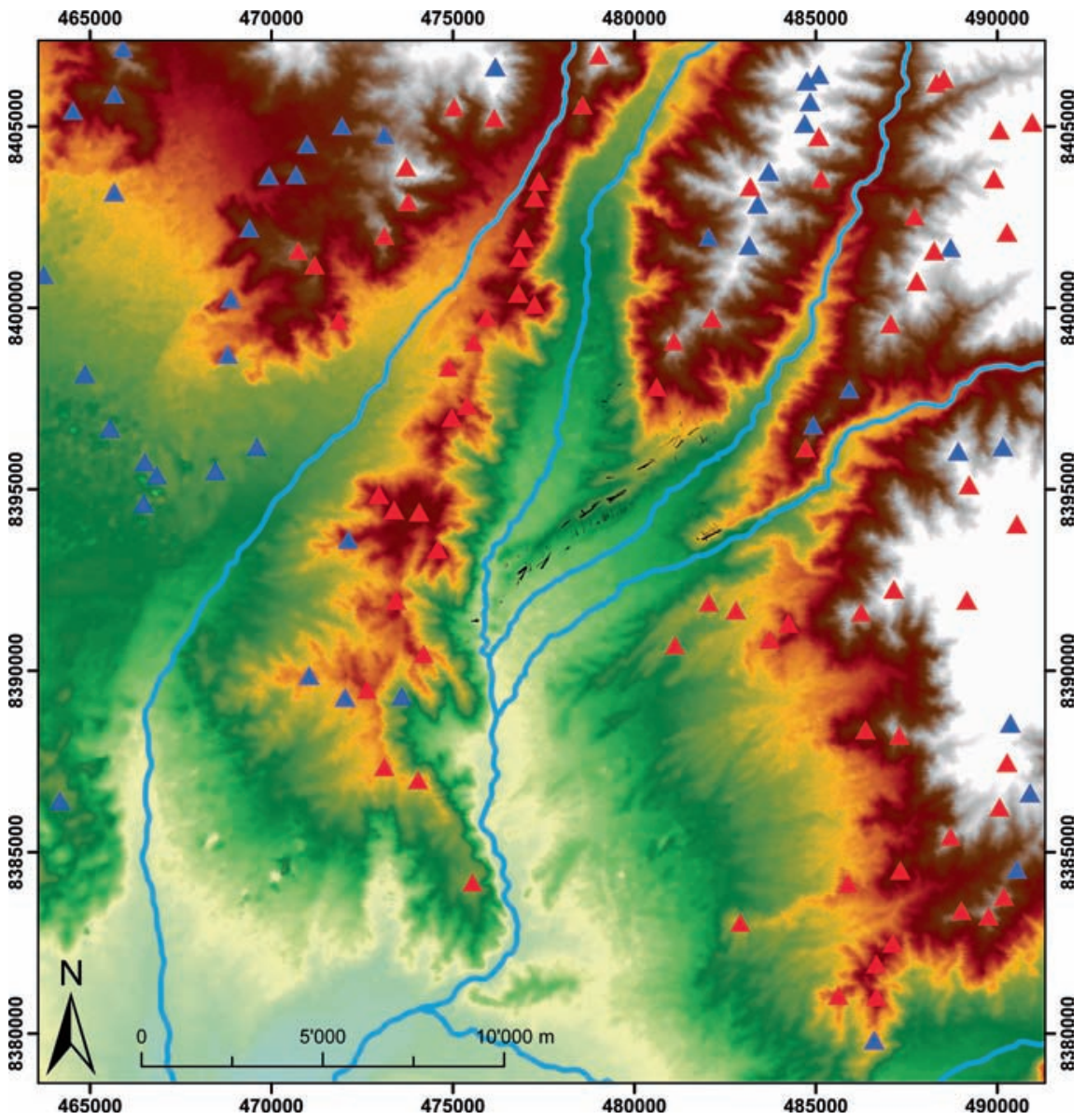

Fig. 19.4 Digital terrain model covering the study area around Palpa. Geoglyphs are shown in black near the centre of the map. Peaks determined in Landserf that are visible from the geoglyphs are shown as red triangles, whereas blue triangles represent peaks outside the area covered by the multiple viewshed. (Coordinates: UTM 18 S, WGS 84)

However, these results could not be taken at face value. As the intersections of axes of orientation and local maxima were calculated in 2D space, possible terrain obstacles blocking the view between both features in 3D space were not considered and had to be factored in in a next step. We therefore calculated lines of sight along the 28 axes using our own software which was adapted to single line of sight calculation (Lambers and Sauerbier 2006). The programme considers earth curvature and refraction. For each axis we defined its intersection with the far end of the respective geoglyph as seen from the peak as the observer's point in order to simulate a view of an observer along the geoglyph towards the horizon. Furthermore, to achieve more realistic results, we 


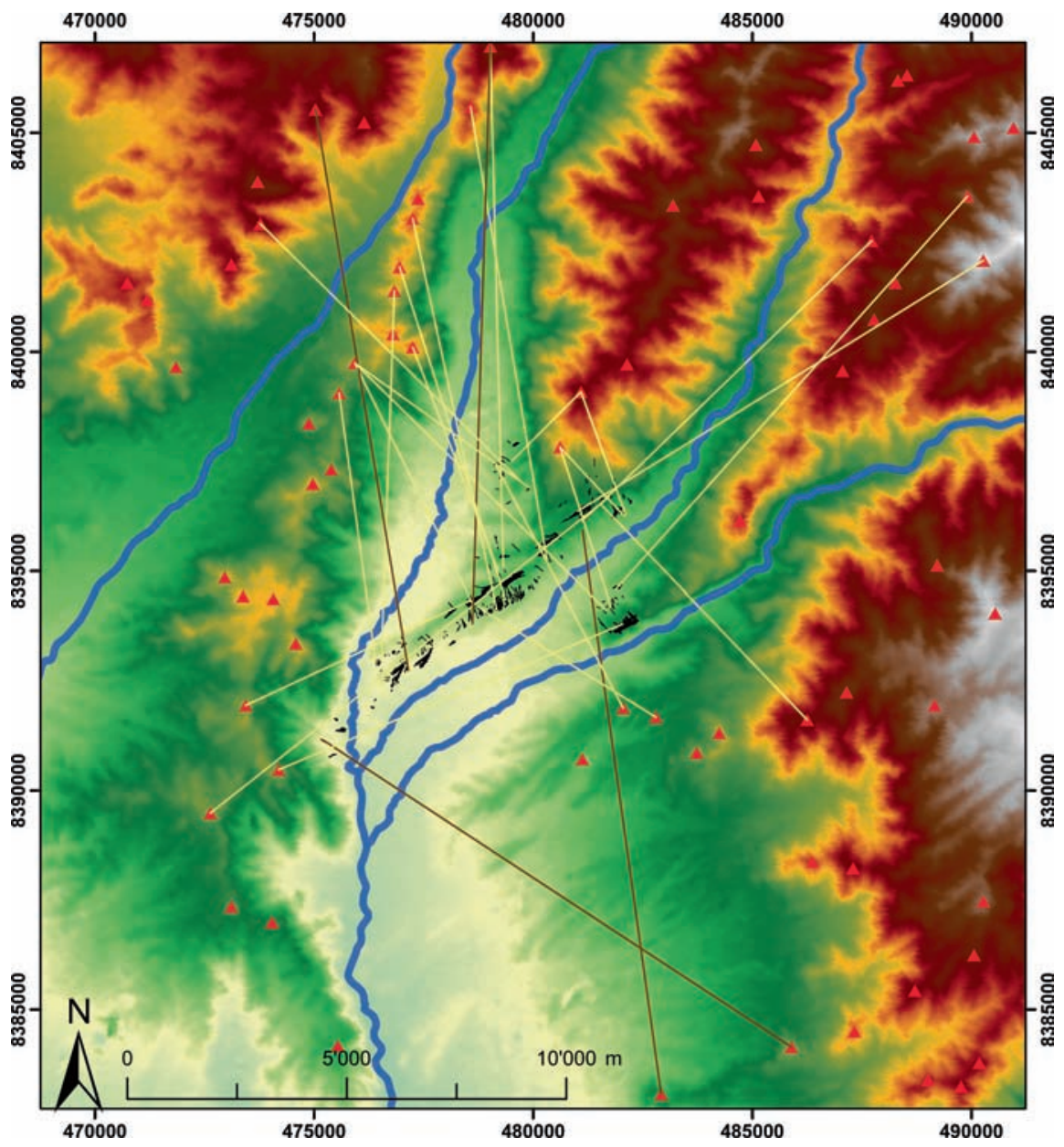

Fig. 19.5 Axes of orientation intersecting mountain peaks. Lines in bright yellow indicate continuous lines of sight, whereas dark green lines break off shortly before reaching the peak but are still regarded as indicating visibility according to the conditions formulated by us (cp. Fig. 19.6). (Coordinates: UTM 18 S, WGS 84)

considered that lines of sight between geoglyph and peaks needed not necessarily end exactly at the respective peak, but might end shortly before reaching that point. This is because when viewing a hill or mountain from the valley, one often gets the visual impression of seeing its peak, even though the actual highest point might be hidden behind the uppermost portion of the slope. In order to model this, we regarded visibility to be given even if the line of sight were blocked by the DTM below the actual peak, the condition being that the remaining distance to the peak must be covered by ascending terrain (Fig. 19.6). 


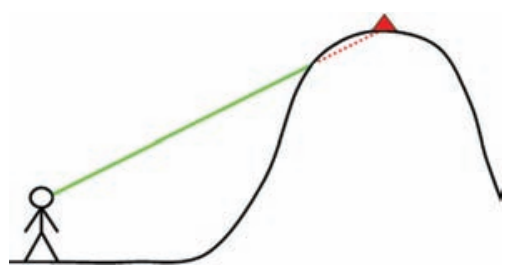

Fig. 19.6 Example of a LoS (green line) not reaching the actual mountain top (red triangle) but stopping shortly before. In this case, because the remaining distance to the mountain top is covered by ascending terrain, the mountain peak is assumed to be visible regardless (cp. Fig. 19.5)

This calculation yielded the following results. Out of the 28 axes of orientation that intersect peaks, continuous lines of sight between geoglyphs and peaks exist in 24 cases. In the remaining 4 cases, the lines of sight end shortly before reaching the peak, with the remaining distance covered by ascending terrain, meaning that visibility was given as well according to our conditions (Figs. 19.5 and 19.6). The preliminary numbers given above were thus confirmed.

In sum, 28 geoglyphs out of the partial sample of 421 geoglyphs with straight sections considered in this study, and out of a total sample of 639 geoglyphs can be regarded as being oriented towards mountain peaks. This corresponds to $6.7 \%$ of the partial sample and $4.4 \%$ of the total sample, respectively.

\subsection{Discussion: Geoglyph Visibility and Orientation}

The question underlying our spatial investigation of the Palpa geoglyphs was in which way a consideration of the surrounding landscape can contribute to an understanding of the geoglyphs. Only in GIS could this kind of contextual analysis be undertaken in a systematic way.

Concerning geoglyph visibility, we extended proven methods of cumulative viewshed calculation to determine if the variable visibility had an impact on the location of the geoglyphs. The results of our study indicate that this was indeed the case. Places with good visibility were apparently preferred for the construction of geoglyphs over places with low visibility, even though the latter were not completely avoided. This allows us to interpret the abundant traces of group activity recorded on geoglyph sites in the framework of rites performed to be seen by others. Geoglyphs can thus be understood as stages rather than images. On these stages social groups acted and interacted, and spectators in the valleys and on other geoglyph sites were able to watch and observe. This visual interaction may have played a role in the differentiation of social groups within Nasca society. This finding does not in itself provide an explanation of the geoglyphs or the acts performed on them, however, it allows important clues about the social context of geoglyph-related activities. 
The material remains of the activities performed on geoglyphs provide hints as to the ideas and concepts that motivated these acts. Objects such as Spondylus shells, crawfish, and field crops placed on stone platforms indicate a strong concern for water, irrigation, and fertility. The assumption that mountains may have been regarded as sources of water led us to explore the orientation of geoglyphs towards this type of prominent landscape feature. Although parts of this analysis could be performed in standard GIS software such as ArcGIS, we had to develop additional tools for the specific requirements of our analysis, namely for the calculation of the axes of orientation of the geoglyphs and their intersection with mountain peaks. The question was whether prominent landscape features such as mountain peaks were visually integrated into the geoglyph scenery by linear geoglyphs pointing towards them.

Although less clear than the visibility study, the results of our orientation study do allow us a preliminary answer to this question. The fact that only about $4-7 \%$ of the geoglyphs of Palpa (depending on the initial sample) are apparently oriented towards mountain peaks clearly shows that such orientations were not a major concern to the people who built the geoglyphs. In fact, the numbers are quite low, considering that the pampas on which the Palpa geoglyphs are located are surrounded by ranges of mountains and hills on three sides (to the north, east, and west). Clearly, orientation towards mountain peaks was not a major ordering principle during geoglyph construction. This conclusion is supported by the observation that the above-mentioned 28 geoglyphs do not include any of the visually predominant geoglyphs of the study area, that is, the large trapezoids and the wide straight lines on the flat plateaus. Rather, most of the 28 geoglyphs are minor elements of large complexes in which the more prominent geoglyphs are apparently not oriented towards mountain peaks.

In spite of these rather clear findings, the fact that 4 out of 23 peaks are intersected by more than one axis of orientation, one of them even by three axes, leaves the possibility open that some geoglyphs were indeed deliberately oriented towards specific mountain tops. We do not know if the corresponding mountains show any special features, for example, archaeological remains on their peaks such as those recorded on Cerro Blanco in the Nasca valley (Reinhard 1996). But even without such features these mountains may once have had a special meaning now concealed to us. This requires further investigation before definite conclusions can be drawn.

In sum, although a small number of geoglyphs may have been deliberately oriented towards mountain peaks, such an orientation cannnot be regarded as a major ordering principle underlying geoglyph placement and construction. In this regard our conclusions are remarkably similar to those of archaeoastronomical studies of the Nasca geoglyphs: although there are indications that some astronomical alignments may have existed, the majority of geoglyph orientations cannot be explained in this way (Aveni 1990). The analogy goes even further. Just as the negative results of archaeoastronomical studies do not mean that astronomy was not important in Nasca society, the negative results of our 
study do not imply that mountain worship did not exist. We can only show that, if it existed, it did not become manifest in consistent spatial relationships between geoglyphs and mountains. It should be noted in this context that our workflow of calculations can also be used for an archaeoastronomical study of the Palpa geoglyphs, which was not a part of our project.

A general conclusion from our spatial study is that the landscape played an important role in the conceptual framework of the geoglyph phenomenon. In the majority of cases, the locations where geoglyphs were placed were carefully chosen such that the acts to be performed on them would be well visible. People on other geoglyph sites, but also down in the valleys, were this way integrated into the activity on the geoglyphs. Visual links to other landscape features, on the other hand, may have been less important, although this requires further studies. The landscape was apparently perceived as a whole, not divided into fertile valleys and barren desert as today. To consider this spatial context is as important when trying to interpret the geoglyphs as the temporal and cultural context.

\subsection{Conclusions: GIS Applications in Archaeology}

Apart from answering questions about geoglyph function and meaning, our study of geoglyph visibility and orientation also illustrates some of the chances and limitations of GIS applications in archaeology.

As mentioned above, GIS was not originally designed for archaeological applications. In our project this became apparent at many stages. Although software packages such as ArcGIS provide a powerful toolbox for a wide variety of analyses, many of the requirements resulting from our research problem were not met by them. In the study described here, standard tools for LoS calculation were not sufficient for our purposes, and tools for the calculation of the centre of gravity of the geoglyphs and the axis of orientation running through them had to be newly developed. There are still very few readymade GIS models and methods available for archaeological investigations. Thus, archaeological GIS applications have to confront many practical problems.

A more general problem stems from the fact that the digital data analysed in GIS are always a simplification of the real world. There are many potential sources of error in the long process of measurements and calculations that led to the results of our study. These range from the accuracy and resolution of the DTM to the accuracy of the geoglyph mapping, the identification of mountain peaks, and finally the various methods of calculation. An unfavourable accumulation of these errors may lead to false results. This is indeed the case for at least one of the 28 axes of orientation that is supposed to hint at a mountain peak which, as we know for certain from our fieldwork, is in fact not visible from the corresponding geoglyph. Such discrepancies between the real world 
and its virtual representation modelled in GIS are common but rarely considered in detail. We do not regard this problem as questioning our overall results, but we do not want it to go unmentioned either.

In spite of these issues, we were able to use GIS to shed light on important spatial relationships between the geoglyphs and the landscape in which they are situated. We used spatial proxies such as terrain elevation, Euclidian distance, azimuth angles, and the like to investigate the ways in which the landscape of Palpa was seen and perceived by the people who built and used the geoglyphs. GIS allowed us to address this question, which had so far been discussed mainly in impressionistic terms, in a systematic way and to draw well-founded conclusions concerning the cultural and social context of the geoglyphs. In this regard our study answers the concerns raised in the first section about the general usability of GIS in archaeological research. In our view the capabilities of GIS to spatially analyse archaeological features, thereby contributing to their interpretation, are far from exhausted.

Nevertheless, the further one explores the potential of GIS to address archaeological research problems, the clearer become its conceptual limitations. All GIS-based studies have to be undertaken within the narrow confines of the concept of physical Cartesian space and the need to quantify and georeference all information. To deal with qualitative, subjective, fragmented, or ambiguous information concerning cultural, social, and ideological aspects of the history of ancient societies within these conceptual limitations clearly remains a challenge. In this sense, GIS should not be regarded as a panacea for archaeological research, but rather as a powerful tool to systematically test carefully formulated hypotheses regarding spatial aspects of cultural history. 